\title{
Les acides gras hydroxylés : agro-tensioactifs aux propriétés moussantes originales
}

\author{
Anne-Laure FAMEAU ${ }^{1}$ \\ Fabrice COUSIN ${ }^{2}$ \\ Arnaud SAINT-JALMES ${ }^{3}$ \\ François BOUE ${ }^{2}$ \\ Jean-Paul DOULIEZ ${ }^{4}$ \\ 1 UR1268 Biopolymères Interactions \\ Assemblages, \\ INRA Nantes, \\ rue de la Géraudière, \\ 44316 Nantes, \\ France \\ <anne-laure.fameau@nantes.inra.fr> \\ 2 Laboratoire Léon-Brillouin, \\ CEA Saclay, \\ 91191 Gif-sur-Yvette cedex, \\ France \\ 3 Institut Physique de Rennes, \\ Université Rennes 1, \\ 35000 Rennes, \\ France \\ ${ }^{4}$ UMR 1332 Biologie du fruit et \\ Pathologie, \\ INRA, \\ 33140 Villenave d'Ornon, \\ France
}

Article reçu le 8 octobre 2012

Accepté le 29 octobre 2012

Parmi les grands enjeux actuels de la recherche en chimie verte, la valorisation d'agro-ressources reste un véritable défi scientifique, technologique et environnemental. À I'heure actuelle, la dépendance des industries chimique, pharmaceutique et cosmétique aux ressources fossiles reste très importante. L'enjeu pour ces industries est donc de trouver de nouvelles matières premières, renouvelables, non toxiques et biodégradables, issues d'agro-ressources. Les agro-produits recherchés doivent donc

\begin{abstract}
Hydroxylated fatty acids: Agro-based surfactants with original foaming properties

Surfactants, which are molecules bearing a hydrophobic part and a hydrophilic part, are widely used in many daily life products (detergent, cosmetic, pharmaceutical. . .) because of their foaming properties. Indeed, they spontaneously go to the interface between air and water, which stabilizes the thin water films surrounding air bubbles to obtain foams. Currently, since the most used surfactants come from derivatives from petroleum-based products, their replacement by biomolecules is a major issue in the current context of sustainable development and green chemistry. This could promote the use of molecules from agricultural resources, such as hydroxyl fatty acids that can be extracted from oil or from the tegument of fruits and vegetables, as new agro-based surfactants. Here, we present recent research on the surfactant properties of the 12-hydroxy stearic acid coming from the castor oil. This fatty acid has very advantageous foaming properties compared to conventional synthetic surfactant systems: it enables the production of foams which are at the same time very abundant and, remarkably, stables over a span lasting more than 6 months. Moreover, these foams are thermoresponsive because the foam stability can be easily modulated by a simple modification of the temperature.
\end{abstract}

Key words: surfactant, fatty acid, supramolecular assembly, interface, foam

présenter des spécificités au moins équivalentes, voire améliorées, à celles des dérivés pétrochimiques en termes de fonctionnalités, sans être nocifs d'un point de vue environnemental. Ceci permettra ainsi de répondre, d'une part, au problème de la raréfaction des ressources $d$ 'origine pétrolière et, $d$ 'autre part, à l'attente des consommateurs qui sont à la recherche de produits plus respectueux de l'environnement.

Les tensioactifs ou agents de surface sont des molécules amphiphiles possédant une partie hydrophile et une partie hydrophobe. Ils sont utilisés dans un grand nombre de produits de notre quotidien du fait de leurs propriétés moussantes (détergents, cosmétiques, pharmaceutiques...). Ils se placent en effet spontanément à l'interface entre l'eau et I'air, ce qui permet de stabiliser les films d'eau très fins autour des bulles d'air pour former des mousses. La plupart des tensioactifs utilisés actuellement étant issus de dérivés pétroliers, il faut chercher à renforcer la part des agrotensioactifs d'origine végétale dans les produits de consommation courante. Une voie envisageable prometteuse est I'utilisation d'acides gras à longues chaînes et hydroxylés. Ces acides gras à longues chaînes sont à I'heure actuelle très peu valorisés alors qu'ils peuvent être extraits des plantes, notamment des téguments des fruits et des légumes, et qu'ils représentent une biomasse importante des industries de la conserverie. Depuis plusieurs siècles, les acides gras sont pourtant utilisés comme substance lavante et nettoyante lorsqu'ils sont à courtes chaînes et demeurent encore très utilisés de nos jours dans les secteurs de la détergence et de la cosmétique. L'exemple le plus célèbre est le savon de Marseille qui est obtenu par saponifica-

Pour citer cet article : Fameau AL, Cousin F, Saint-Jalmes A, Boue F, Douliez JP. Les acides gras hydroxylés : agro-tensioactifs aux propriétés moussantes originales. OCL $2013 ; 20(1): 8-15$. doi : 10.1684/ocl.2012.0491 
tion de l'huile d'olive. La limitation de l'utilisation d'acides gras à longues chaînes dans ces domaines industriels vient du fait que ces biomolécules sont insolubles dans l'eau à température ambiante (Cistola et al., 1986). Leur valorisation passe donc par une nécessaire étape de dispersion dans l'eau. Des études récentes menées par plusieurs groupes de recherche ont montré que cette étape peut être réalisée simplement à température ambiante en utilisant des sels spécifiques (Zana, 2004). Ces études ouvrent ainsi la voie à la valorisation de ces sels d'acides gras à longues chaînes comme nouveaux agrotensioactifs.

Les acides gras à longues chaînes et hydroxylés utilisés en tant que tensioactifs ont donc un fort potentiel économique puisque les agro-tensioactifs, issus de matières premières renouvelables, jouissent d'une très bonne image auprès du grand public, due à la fois à leur faible toxicité, leur faible écotoxicité et à leur faible innocuité pour la santé humaine (Vandeputte, 2012). En pratique, il faut trouver le sel adéquat permettant la dispersion aqueuse de ces acides gras pour pouvoir ensuite les utiliser en tant qu'agent moussant. Nous présentons ici des recherches récentes concernant les propriétés tensioactives de l'acide gras 12-hydroxystéarique issu de I'huile de ricin. Après une brève description des mécanismes d'autoassemblage des tensioactifs et de formation d'une mousse, nous allons montrer comment, à partir de cet acide gras hydroxylé à longue chaîne, il est possible d'obtenir des mousses aux propriétés nettement supérieures à celles formées à partir de tensioactifs classiques synthétiques, puisqu'elles ont une stabilité exceptionnelle à température ambiante supérieure à six mois et que ce sont les premières mousses thermosensibles.

\section{Dispersion et agrégation des acides gras à longues chaînes en solution}

Un tensioactif est une molécule amphiphile qui possède une partie hydrophile et une partie hydrophobe. C'est l'antagonisme de cette structure qui lui confère des propriétés remarquables au niveau du pouvoir émulsionnant, pouvoir moussant ou pouvoir mouillant. Les tensioactifs entrent dans la formulation des produits détergents et des produits cosmétiques comme les shampoings, crèmes, maquillage... Lorsque I'on disperse en solution aqueuse des molécules tensioactives, elles vont chercher à minimiser les contacts entre leur partie hydrophobe et l'eau. Elles vont donc s'adsorber préférentiellement aux interfaces, ce qui leur est énergétiquement favorable. Ceci entraîne une diminution de la tension interfaciale entre l'air et l'eau. C'est la raison pour laquelle ces molécules sont appelées "tensioactives " ou " agents de surface ". Lorsque l'interface du système est saturée en tensioactifs et que l'on augmente la concentration en solution, les monomères en excès étant contraints de rester dans le volume, ils s'auto-organisent spontanément pour former des assemblages supramoléculaires de formes variées leur permettant de minimiser les contacts entre leurs parties hydrophobes et les molécules d'eau. Cette autoorganisation apparaît au-delà d'une concentration seuil appelée " concentration d'agrégation critique " (CAC). Des considérations géométriques simples permettent de déterminer a priori les organisations possibles, en solution aqueuse, d'un tensioactif donné. Elles sont basées sur le lien qui a été établi en 1976 entre la géométrie du tensioactif et la morphologie des assemblages obtenus (Israelachvili, 1992) et dont le paramètre-clé est le paramètre d'empilement $p$ (figure 1). II correspond au rapport entre le volume de la tête polaire du tensioactif $v$ et le volume correspondant à l'aire de cette dernière $a_{o}$ multipliée par la longueur critique $I_{c}$ de la chaîne hydrophobe :

$$
\mathrm{p}=\mathrm{v} /\left(\mathrm{a}_{0} * \mathrm{I}_{\mathrm{c}}\right)
$$

À partir de ce paramètre d'empilement, la structure des assemblages formés en solution peut être facilement déduite de la géométrie du tensioactif. Lorsque le volume de la tête hydrophile du tensioactif est prépondérant par rapport à la chaîne hydrophobe $(p<1 / 3)$, on obtient des micelles. Lorsque le volume occupé par la partie hydrophobe est égal ou supérieur à celui de la partie hydrophile, des vésicules $(1 / 3<p<1 / 2)$ sont obtenues. Pour $p=1$, on observe des phases lamellaires et pour $p>1$ des phases inversées (figure 1). Le type $d^{\prime}$ 'assemblage formé par les tensioactifs en solution varie en fonction de la structure intrinsèque du tensioactif (nature de la partie hydrophile, nature de la partie hydrophobe) mais également en fonction de paramètres extrinsèques (solvant, température, salinité, concentration) qui modifient des interactions attractives et répulsives entre molécules tensioactives.

Les acides gras sont des composants présents en abondance dans les graisses

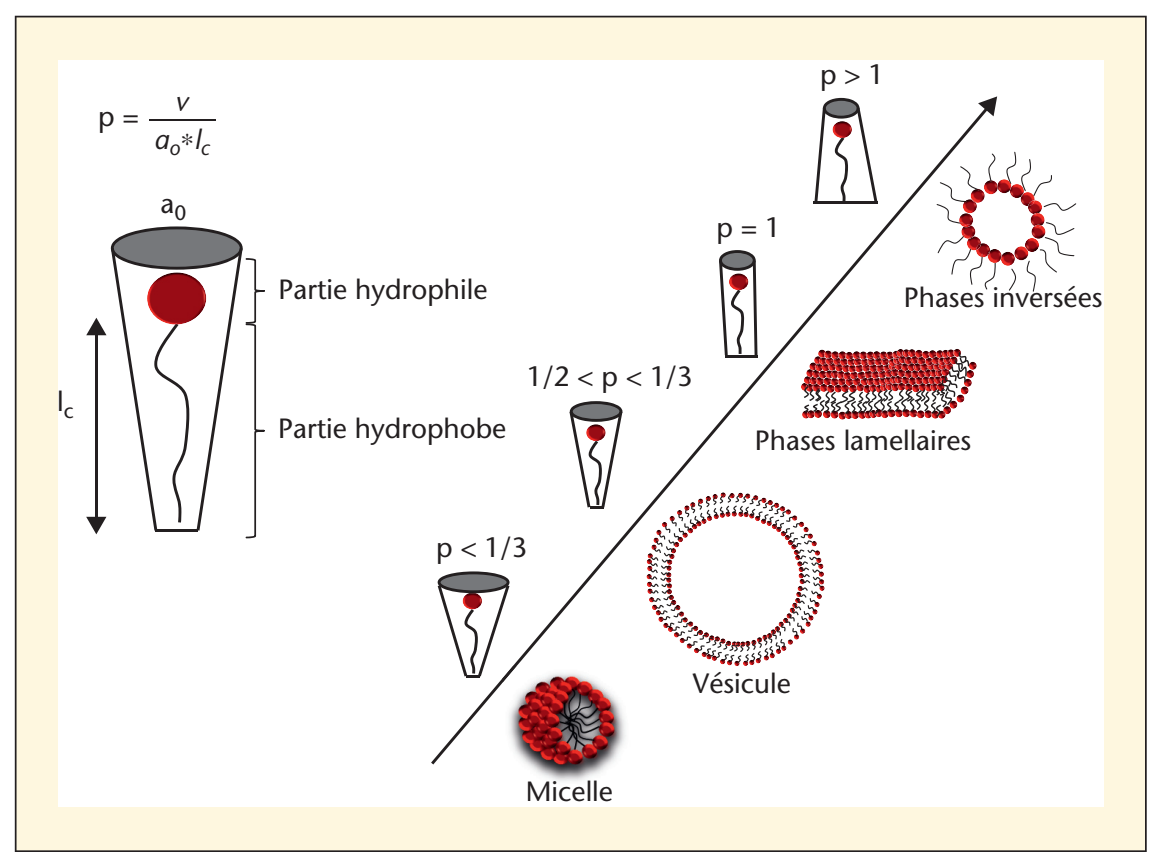

Figure 1. Assemblages supramoléculaires formés en fonction de la géométrie du tensioactif. 
animales et végétales. Ce sont des molécules possédant une tête hydrophile (groupement carboxylique) et une queue hydrophobe (chaîne alkyle), elles sont donc naturellement tensioactives (Karleskind, 1992). La tête hydrophile des acides gras qui porte la fonction carboxylique $\mathrm{COOH}$ est polaire dans l'eau et lorsque la fonction est dissociée en $\mathrm{COO}^{-}$la polarité augmente. La chaîne carbonée étant par contre apolaire, la solubilité des acides gras dans l'eau diminue avec l'augmentation du nombre de carbones. A partir de 5 carbones, les acides gras sont insolubles sous forme de monomères et s'organisent, soit aux interfaces, soit sous forme $\mathrm{d}^{\prime}$ assemblages ou de cristaux en solution (McBain et al., 1920). Les acides gras à longues chaînes (nombre de carbones $>12$ ) ne peuvent pas s'autoassembler dans l'eau à température ambiante car ils cristallisent, ce qui empêche leur utilisation en tant que tensioactif. L'enjeu est donc d'arriver à disperser ces acides gras à longues chaînes et hydroxylés pour pouvoir ensuite exploiter leurs propriétés tensioactives. Une stratégie permettant la dispersion des acides gras à longues chaînes est l'utilisation de contre-ions chargés positivement permettant la formation de sels d'acides gras. L'ajout du contre-ion va permettre la dissociation du groupement carboxylique $\mathrm{COOH}$ de la tête hydrophile de l'acide gras en groupement carboxylate $\mathrm{COO}^{-}$ chargé. Le caractère polaire de l'acide gras est alors augmenté car l'adjonction de charges entraîne une meilleure solvatation des têtes hydrophiles. Les sels d'acides gras les plus connus sont les sels de sodium ou de potassium. Néanmoins, I'utilisation de ces sels n'est pas optimale pour les acides gras à longues chaînes car ils restent faiblement solubles dans l'eau à température ambiante. Récemment, il a été montré que la solubilité de l'acide gras pouvait encore être améliorée de façon significative à température ambiante en substituant le contre-ion alcalin par une hydroxyalkylamine (tel que la lysine ou la choline). Ceci provient de la réaction acido-basique entre la fonction acide carboxylique $\left(\mathrm{COOH} / \mathrm{COO}^{-}\right)$de l'acide gras et I'amine basique $\left(\mathrm{NH}_{2}{ }^{+} / \mathrm{NH}_{3}{ }^{+}\right)$ (Klein et al., 2008; Novales et al., 2010). Une fois dispersés par cette méthode, les acides gras peuvent $s^{\prime}$ auto-assembler en solution et donc être utilisés comme agro-tensioactifs pour produire des mousses (Fameau et al., 2011c).

\section{Définition et propriétés des mousses}

Une mousse est un ensemble de bulles de gaz dispersées dans une phase continue liquide qui contient les agents stabilisant l'interface air/eau. Une façon simple de produire une mousse est I'agitation manuelle d'une solution contenant des molécules tensioactives, ce qui permet d'introduire des bulles de gaz dans le liquide. Pour étudier plus finement les propriétés des mousses, il existe une méthode alternative de production dite "par bullage", qui consiste à former une mousse par injection de gaz dans une solution à travers des orifices de taille et de morphologie contrôlées (tube capillaire, disque poreux, etc.) permettant d'obtenir des bulles de taille contrôlée.

La mousse est constituée d'un ensemble de bulles compressées les unes contre les autres et séparées par un réseau continu contenant le liquide (figure 2). $C^{\prime}$ est un système multi-échelles avec différents niveaux d'organisation. Du plus petit au plus grand, on trouve à l'échelle nanométrique l'interface entre I'air et la phase liquide stabilisée par les tensioactifs. II y a ensuite le film mince qui correspond à deux interfaces air/eau en regard dont l'épaisseur typique est de quelques dizaines de $\mathrm{nm}$ : il s'agit de la zone de contact entre deux facettes de bulles. L'échelle au-dessus du film mince correspond au canal liquide formé à la zone de jonction de trois films minces : ce canal s'appelle " bords de Plateau " et fait quelques dizaines de microns. Enfin, aux échelles les plus grandes, quatre bords de Plateau se rejoignent pour former un tétraèdre dans ce que I'on appelle généralement un « nœud " (Cantat et al., 2010).

La principale caractéristique d'une mousse est sa stabilité puisque ce sont des systèmes intrinsèquement instables d'un point de vue thermodynamique du fait que l'énergie libre associée à la mousse est supérieure à celle du système liquide-gaz séparé. Ainsi, dès la fin même de leur formation, les mousses évoluent afin de minimiser cette énergie libre, ce qui conduit au final à leur destruction et au retour à l'état d'équilibre biphasique gaz/liquide (Weaire et al., 1999). La cinétique de destruction est très variable d'un système à l'autre. Certaines mousses ont une durée de vie de l'ordre de quelques secondes (le champagne), de l'ordre de quelques heures (la mousse de bière) ou de quelques jours (mousse au chocolat) (Saint-Jalmes et al., 2002). La mousse se déstabilise sous l'effet de trois principaux processus irréversibles (figure 3 ) :

- le premier mode de déstabilisation est le drainage gravitationnel. En effet, sous I'action de la gravité, le liquide s'écoule dans la mousse du haut vers le bas asséchant irréversiblement la mousse (Cervantes-Martinez et al., 2005) ;

- le second mécanisme de déstabilisation est le mûrissement. II provient de la différence de pression existant entre deux bulles $A$ et $B$ de rayons différents de pressions respectives $\mathrm{P}_{\mathrm{A}}$ et $\mathrm{P}_{\mathrm{B}}$. Cette différence de pression peut s'écrire à partir de la relation de Laplace, qui fait intervenir les rayons de courbure $\left(R_{A}\right.$ et $R_{B}$ ) des bulles et la tension interfaciale $\gamma$ :

$\Delta P=P_{A}-P_{B}=\gamma\left(\frac{1}{R A}+\frac{1}{R B}\right)$

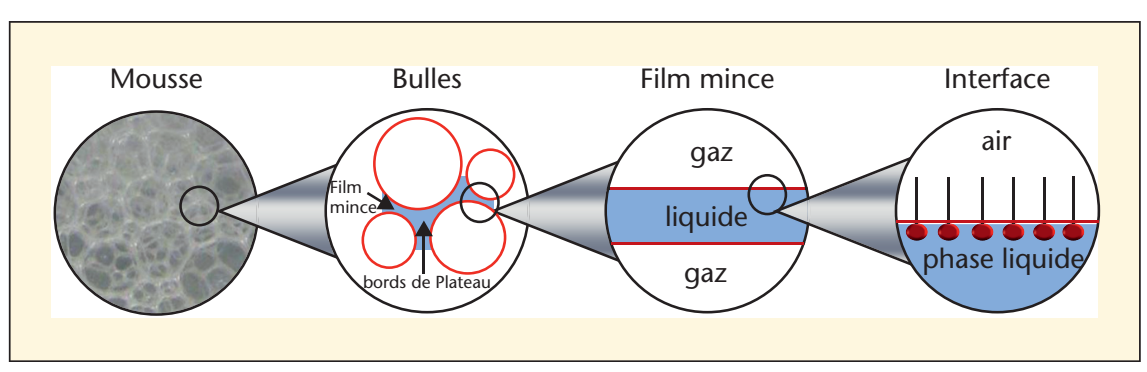

Figure 2. Structure de la mousse aux différentes échelles. 


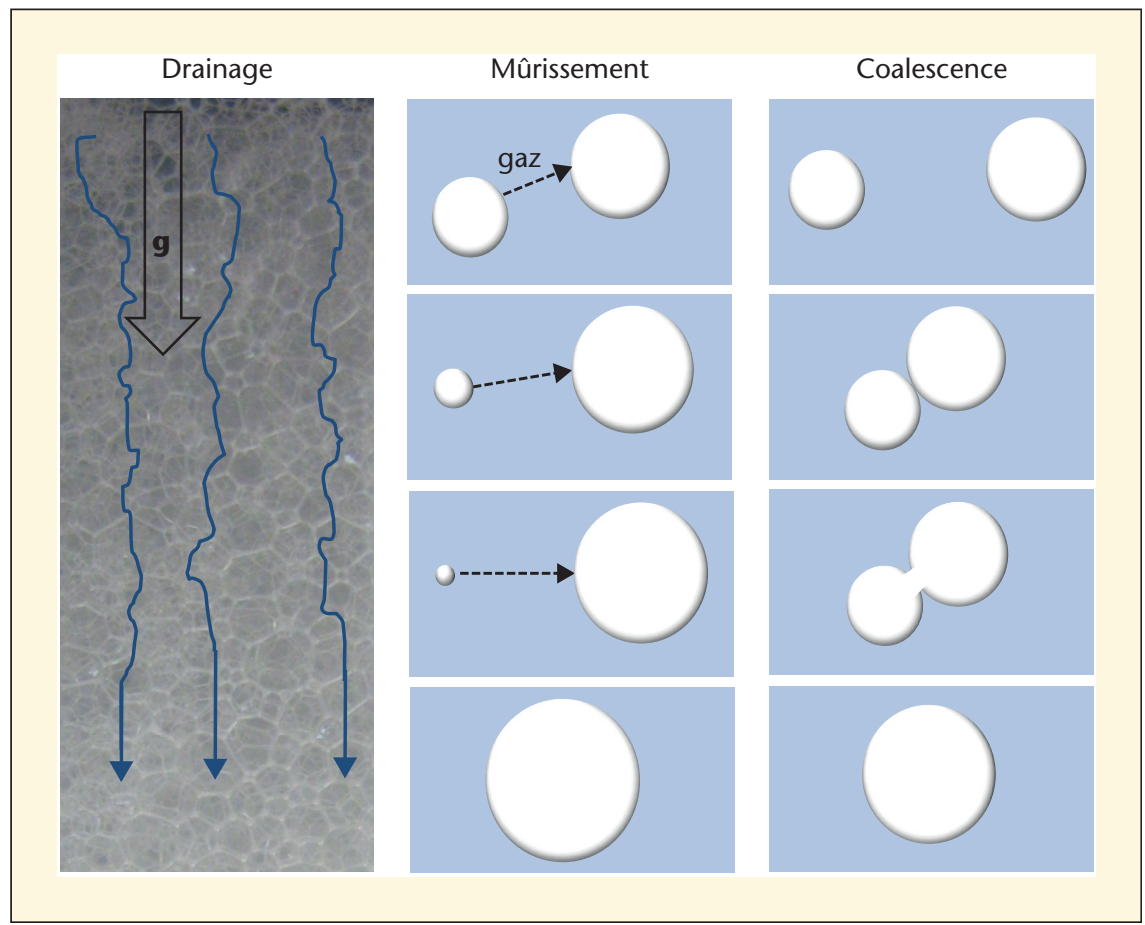

Figure 3. Les trois principaux mécanismes de déstabilisation de la mousse.

La pression est plus forte dans les petites bulles (celles ayant le moins de facettes) que dans les grandes (qui ont un plus grand nombre de facettes), ce qui entraîne un gradient de pression de part et d'autre d'un film et induit une diffusion gazeuse à travers ce film. Ce phénomène fait que les petites bulles se vident progressivement de leur gaz au profit des plus grandes et disparaissent ainsi les unes après les autres. Ainsi, la taille moyenne des bulles restantes augmente progressivement (SaintJalmes, 2006) ;

- le troisième phénomène de déstabilisation est la coalescence. II apparaît lorsque les films séparant deux bulles se cassent. Les deux bulles se réorganisent alors pour n'en former plus qu'une seule. Ceci tend à faire disparaître la mousse. En effet, sous I'action du drainage et du mûrissement, les films séparant les bulles deviennent très minces et très grands en surface de telle sorte qu'ils finissent par se rompre. C'est donc par une succession de ruptures de films que la mousse disparaît. Les films des bulles situés à la surface de la mousse sont les plus susceptibles de rompre puisqu'ils sont les plus fins (sous l'effet du drainage gravitaire). Ainsi, la mousse s'effondre généralement par quantité de mousse obtenue à partir de ces solutions de particules solides est alors très faible (Binks et al., 2005) Au contraire, les tensioactifs classiques dits de faible poids moléculaire migrent et s'adsorbent très rapidement à l'interface permettant l'obtention rapide d'une grande quantité de mousse, mais ils ne forment pas un film solide à l'interface (Binks, 2002). Les phénomènes de coalescence et de mûrissement peuvent donc se produire facilement dans ces mousses et par conséquent la durée de vie de ces mousses n'excède pas quelques dizaines de minutes. Ainsi, jusqu'à présent, l'obtention d'un système simple permettant d'obtenir à la fois une moussabilité optimale (bon agent moussant) et une stabilité exceptionnelle de la mousse (bon stabilisateur) demeure un défi très complexe à relever.

\section{Les tubes d'acides gras 12-hydroxystéarique : un assemblage supramoléculaire aux propriétés originales}

Nos travaux ont porté sur un acide gras particulier, l'acide gras 12-hydroxystéarique provenant de I'hydrogénation de l'acide ricinoléique, lui-même extrait de de I'huile de ricin. Tout d'abord, nous avons montré que les molécules de cet acide gras hydroxylé à longue chaîne s'assemblent en solution aqueuse sous forme de tubes lorsqu'on utilise I'hexanolamine comme contreion pour la dispersion (Douliez et al., 2006). En microscopie, ces tubes d'acides gras apparaissent sous la forme de bâtonnets d'environ $10 \mu \mathrm{m}$ de longueur et $d^{\prime}$ un diamètre externe de $0,6 \mu \mathrm{m}$ à température ambiante. En utilisant la technique de diffusion de neutrons aux petits angles, il a pu être mis en évidence que ces tubes sont composés d'un empilement concentrique de bicouches d'acides gras séparées par de l'eau (figure 4). Ces tubes ressemblent donc à des "poireaux ". Ils conservent leur structure jusqu'à $60{ }^{\circ} \mathrm{C}$, température au-delà de laquelle ils fusionnent pour former alors des micelles sphériques d'acides gras (Fameau et al., 2010). Le diamètre de la micelle est de $4 \mathrm{~nm}$, soit une taille caractéristique environ 1000 fois plus faible que celle du diamètre des tubes. Cette transition avec la température entre l'auto-organisation sous forme de tube et I'auto- 


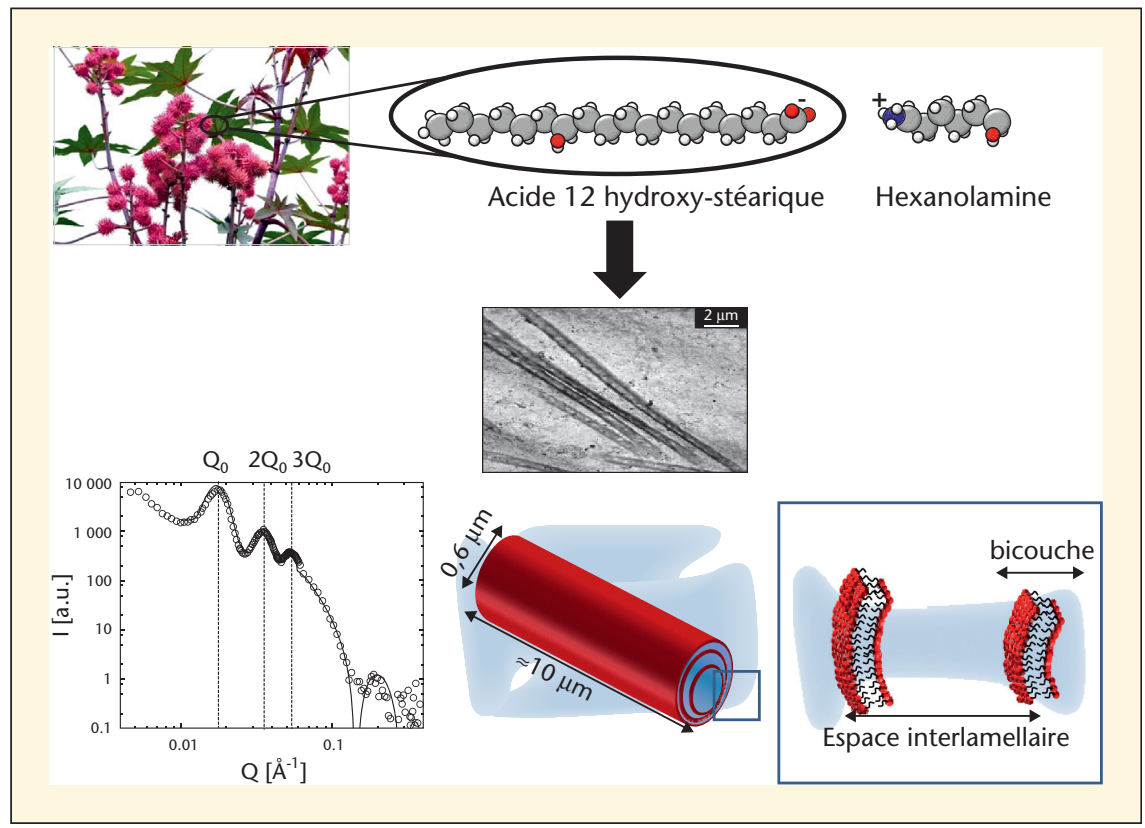

Figure 4. Image de TEM d'une solution de tubes d'acide gras 12-hydroxy-stéarique avec I'hexanolamine comme contre-ion. Spectre de diffusion de neutrons aux petits angles d'une solution de tubes avec les raies de Braggs montrant l'empilement lamellaire au sein des tubes.

organisation sous forme de micelle est complètement réversible (Fameau et al., 2010).

Par ailleurs, la technique de réflectivité de neutrons a permis de montrer que ces tubes ont la capacité de s'adsorber à l'interface air/eau (figure 5) (Fameau et al., 2011b). Les tubes restent adsorbés à l'interface sous une monocouche d'acides gras jusqu'à $60^{\circ} \mathrm{C}$. Audelà de cette température, les tubes fusionnent sous forme de micelles en solution et l'interface est alors stabilisée seulement par une monocouche d'acides gras. De façon remarquable, lorsque

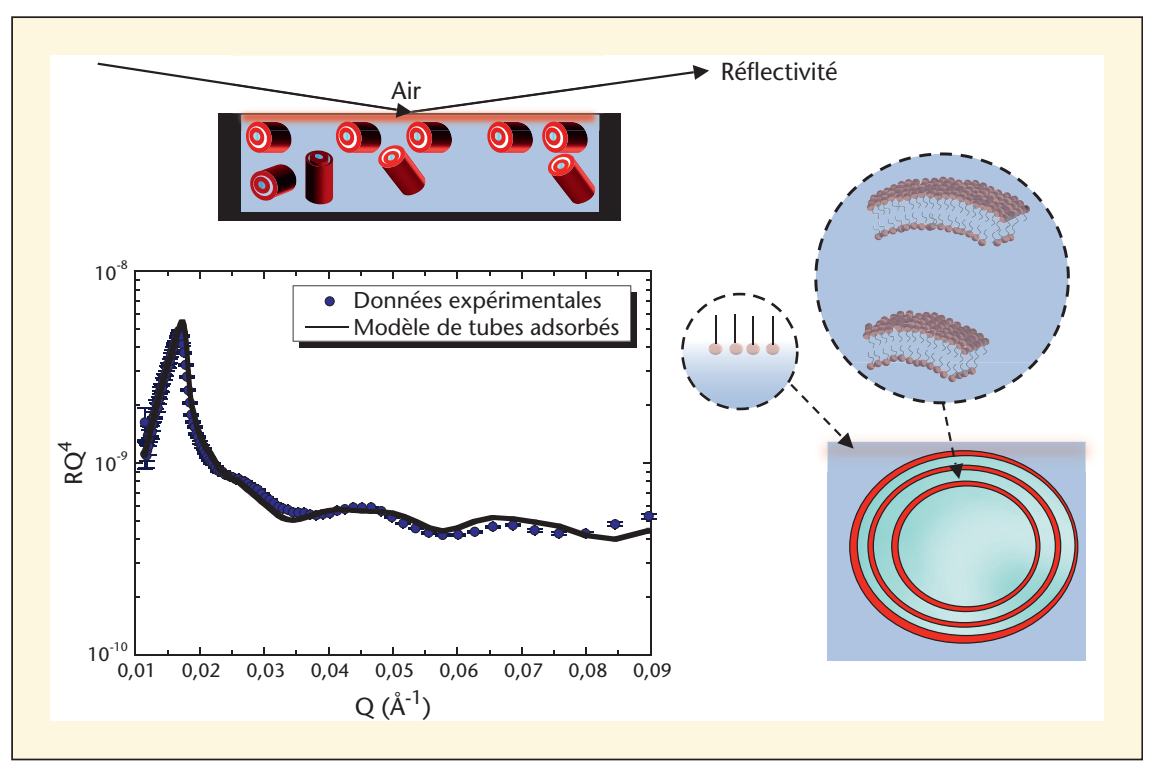

Figure 5. Spectre de réflectivité de neutrons obtenues expérimentalement à l'interface entre l'air et la solution de tubes (en bleu) et comparé au spectre calculé à partir d'un modèle mathématique de tubes adsorbés à l'interface sous une monocouche d'acides gras (en noir).
I'on rediminue la température en dessous de $60{ }^{\circ} \mathrm{C}$, les tubes se reforment à l'interface. Ainsi, la transition entre les tubes et les micelles est complètement réversible à la fois en solution et à l'interface air/eau. Cet assemblage d'acides gras au comportement particulier vis-à-vis de la température avait donc un potentiel intéressant pour produire des mousses thermosensibles.

\section{Mousse stable sur des temps longs à partir des tubes d'acides gras}

Par simple agitation manuelle d'une solution aqueuse de tubes d'acide gras 12-hydroxystéarique, on observe la formation d'une grande quantité de mousse (figure 6). Ces solutions de tubes d'acide gras présentent donc une moussabilité optimale du fait de l'adsorption rapide des monomères $d^{\prime}$ 'acides gras provenant du dépliement de tubes au niveau de l'interface air/eau des bulles. Ceci correspond donc à un comportement normal de tensioactifs décrit précédemment. Par ailleurs, le drainage dans ces mousses apparaît dès les premiers instants après la formation de la mousse, il ralentit ensuite progressivement au bout d'une dizaine de minutes et est ensuite complètement bloqué. Cet arrêt du drainage provient de la présence d'une grande quantité de tubes dans les bords de Plateau ce qui limite l'écoulement du liquide. De façon surprenante, le volume de mousse ainsi que l'aspect de la mousse ne change pas pendant plus de 6 mois (figure 6). Ces mousses sont donc incroyablement stables, contrairement aux tensioactifs classiques dont la stabilité des mousses n'excède pas quelques heures (Fameau et al., 2011d). Ceci provient de la formation d'une monocouche dense et très élastique de monomères $d^{\prime}$ 'acides gras à l'interface air/eau, via des liaisons hydrogène entre les groupements hydroxyles des monomères d'acide gras 12-hydroxystéarique. La présence de cette couche à l'interface permet donc de limiter les phénomènes de mûrissement et de coalescence de la même façon qu'expliquer précédemment dans le cas des mousses à particules. C'est donc la structure chimique particulière de cet acide gras avec la présence d'un groupement hydroxyle en position 12 sur la chaîne carbonée qui permet d'obtenir ce 


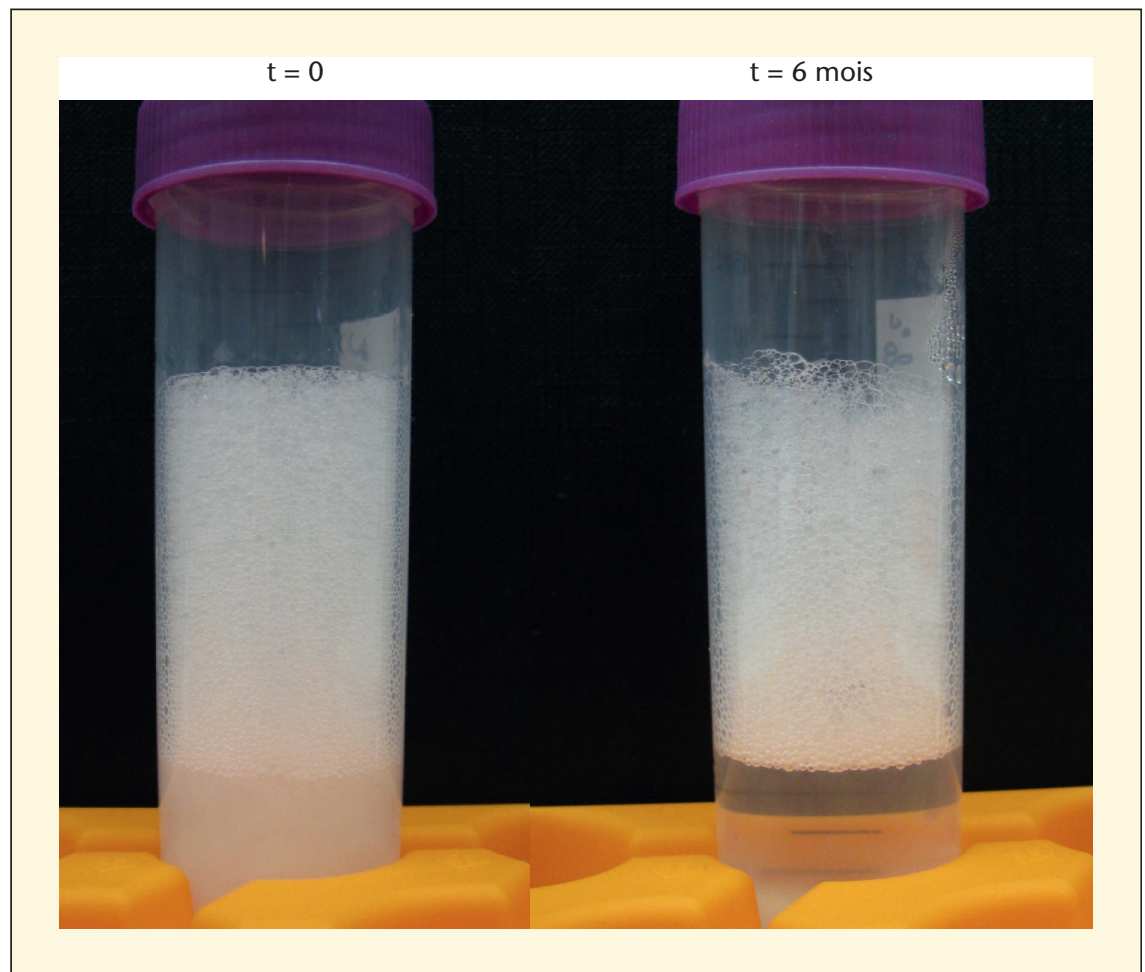

Figure 6. À gauche: photographie d'une mousse obtenue à partir d'une solution de tubes juste après agitation de la solution. À droite: photographie de la même mousse après six mois de conservation à température ambiante.

comportement singulier à l'interface. Pour leur part, les tubes jouent un rôle au niveau des bords de Plateau où ils sont présents en grande quantité, ce qui limite le drainage du liquide. Grâce à ce système particulier de tubes d'acides gras hydroxylés, les trois principaux mécanismes de déstabilisation de la mousse sont ainsi bloqués. Ce système de tubes d'acides gras permet donc d'obtenir une très bonne stabilité dans le temps de la mousse tout en y associant une moussabilité optimale.

\section{Mousse réactive : les premières mousses thermosensibles}

Puisque les tubes d'acides gras jouent un rôle important sur la stabilisation la mousse en limitant le drainage, nous avons ensuite testé l'influence d'une augmentation de la température sur la stabilité de ces mousses au-delà de la température de transition tubes/ micelles en solution. Lorsqu'une mousse est produite par agitation manuelle à $60{ }^{\circ} \mathrm{C}$, température supérieure à cette température de transition, la mousse de phénomènes de déstabilisation de la mousse, provoquant la destruction très rapide de celle-ci. Lorsque le réseau rigide et stable formé par les tubes dans la phase liquide entourant les bulles d'air disparaît, le drainage n'est en effet plus limité car les micelles, de tailles plus petites que les films minces, peuvent s'écouler dans la mousse.

La stabilité des mousses d'acides gras étant très dépendante de la morphologie des assemblages (tubes ou micelles), la transition en température entre ces types d'assemblages peut être mise à profit pour contrôler très facilement et très précisément la stabilisation et/ou la déstabilisation de la mousse. Pour cela, des expériences ont été réalisées en produisant des mousses par bullage de gaz à travers un poreux en verre dans la solution de tubes contenue dans une colonne en verre thermostatée. L'introduction de gaz dans la solution moussante s'arrête dès que le volume fixé de $45 \mathrm{~mL}$ est atteint. Une caméra permet ensuite de suivre l'évolution temporelle de la hauteur, et donc du volume de mousse, pour une température donnée. Ceci est illustré sur la figure 8. Lorsque I'on augmente in situ la température $\mathrm{d}^{\prime}$ une mousse très stable à $20^{\circ} \mathrm{C}$ à I'aide d'une colonne thermostatée afin de franchir le seuil de transition tubes/ micelles à $60^{\circ} \mathrm{C}$, la mousse se déstabilise rapidement et le volume de mousse diminue. Si I'on rediminue ensuite la température à $20^{\circ} \mathrm{C}$, en dessous de cette température seuil, l'évolution du volume de mousse redevient stable en fonction du temps car les tubes se reforment (figure 8). petits, qui a entraîné la reprise des

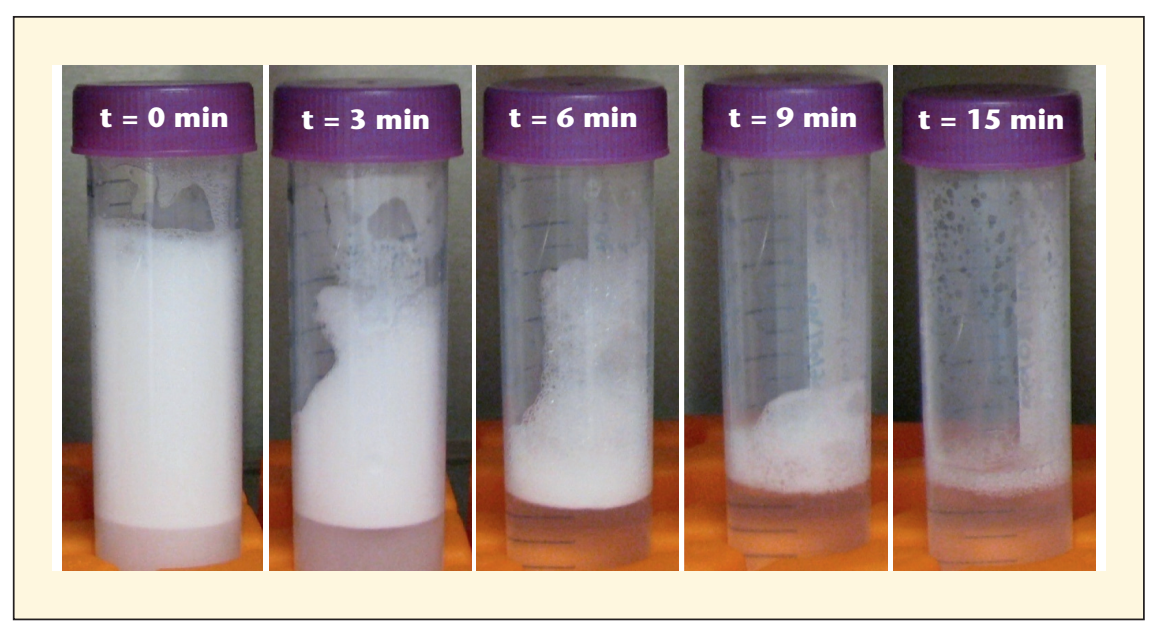

Figure 7. Photographies illustrant la déstabilisation très rapide de la mousse à $60^{\circ} \mathrm{C}$ en fonction du temps en minutes. 


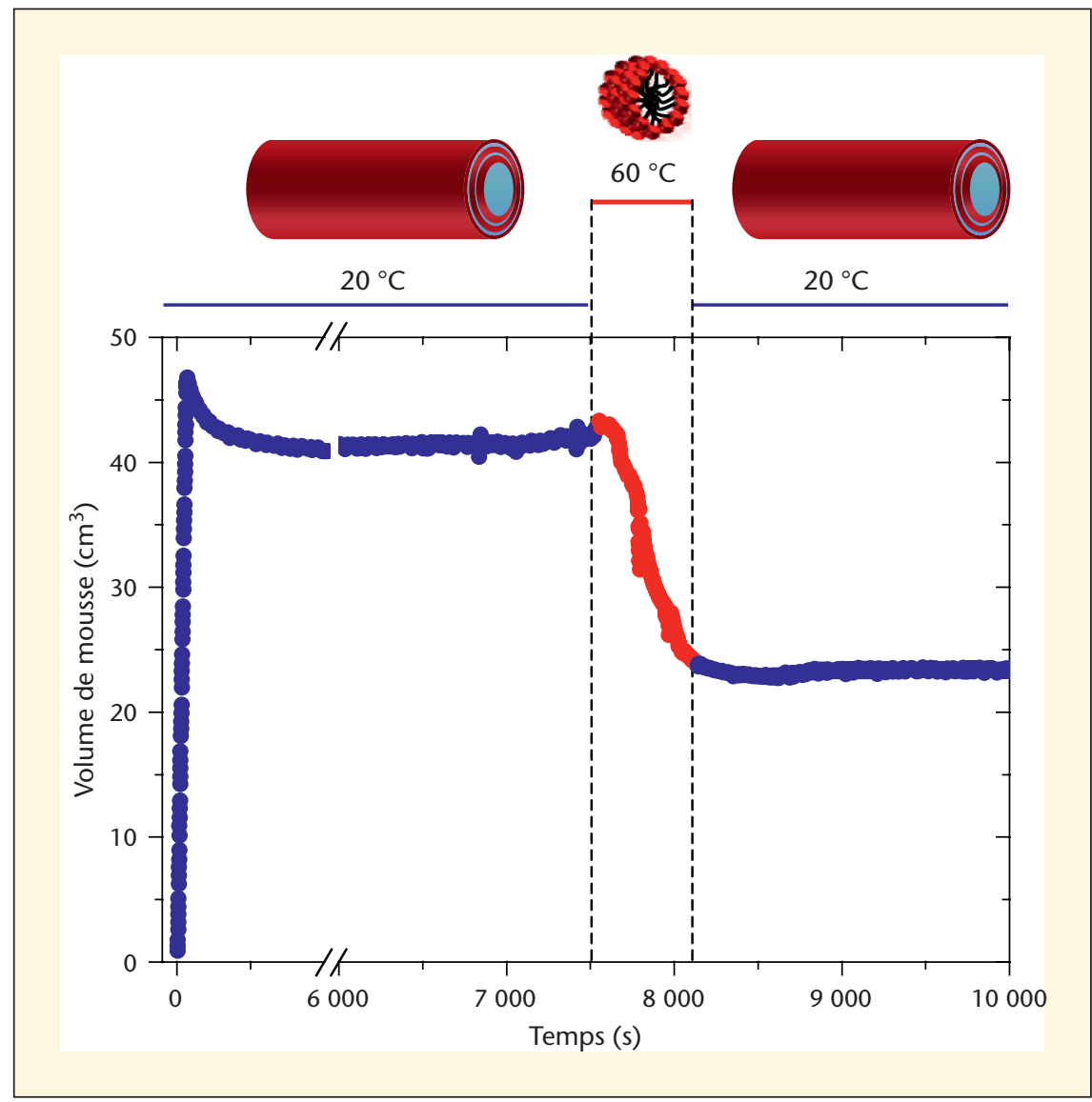

Figure 8. Évolution du volume de mousse en fonction du temps lors de modifications de la température de la mousse qui modifient l'assemblage présent dans la mousse: tubes ou micelles.

Ainsi, en jouant sur la morphologie des assemblages d'acides gras présents dans la mousse (tubes ou micelles) à l'aide de la température, il est possible de moduler très facilement et très précisément la stabilité des mousses. Ce sont donc des mousses qui sont thermosensibles (Fameau et al., 2011d). De plus, en jouant sur le contre-ion utilisé pour disperser l'acide gras 12-hydroxystéarique il est possible de facilement modifier la température de transition entre les tubes et les micelles en solution aqueuse (Fameau et al., 2011a). On peut ainsi moduler aisément la température seuil de déstabilisation des mousses.

\section{Conclusion}

À l'heure actuelle, la valorisation d'agroressources reste un véritable défi scientifique, technologique et environnemental. Ainsi, nos recherches sur les acides gras à longues chaînes et dérivés
- les solutions de tubes ont une moussabilité optimale, c'est-à-dire qu'à partir d'une faible quantité de solution de tubes une quantité importante de mousse est obtenue ;

- les mousses contenant des tubes sont stables sur des temps très longs ;

- les mousses sont thermosensibles car il est possible de les stabiliser/ déstabiliser en jouant sur la température qui modifie l'assemblage présent dans la mousse tubes ou micelles ;

- la température de déstabilisation des mousses peut être modulée sur une large gamme de température (de $20^{\circ} \mathrm{C}$ à $70{ }^{\circ} \mathrm{C}$ ) par le contre-ion utilisé via la température de transition tubes/micelles.

Du fait de leurs spécificités, ces mousses issues d'agro-tensioactifs possèdent plusieurs champs d'application. Ces mousses très stables dans le temps pourront être notamment utilisées pour le développement de cosmétiques ou dans la mise au point de détergents ou de mousses dont on peut contrôler l'action dans le temps en jouant simplement sur la température.

\section{Conflits d'intérêts : aucun}

\section{RÉFÉRENCES}

Binks BP, Horozov TS. Aqueous foams stabilized solely by silica nanoparticles. Angewandte Chemie International Edition 2005 ; 44 : 3722-5.

Binks BP. Particles as surfactants-similarities and differences. Current Opinion in Colloid and Interface Science $2002 ; 7$ : 21-41.

Cantat I, Cohen-Addad S, Elias F, Graner F, Hohler R, Pitois O, Rouyer F, Saint-Jalmes A. Les mousses : structure et dynamique. Paris : Belin, Collection Echelles, 2010.

Carrier V, Colin A. Coalescence in draining foams. Langmuir $2003 ; 19$ : 4535-8.

Cervantes-Martinez A, Saint-Jalmes A, Maldonado A, Langevin D. Effect of cosurfactant on the free-drainage regime of aqueous foams. Journal of Colloid and Interface Science $2005 ; 292$ : 544-7.

Cistola DP, Atkinson D, Hamilton JA, Small DM. Phase-behavior and bilayer properties of fatty-acids-hydrated 1-1 acid soaps. Biochemistry $1986 ; 25: 2804-12$.

Douliez JP, Gaillard C, Navailles L, Nallet F. Novel lipid system forming hollow microtubes at high yields and concentration. Langmuir 2006 ; 22 : 2942-5.

Fameau AL, Houinsou Houssou B, Novales B, Navailles L, Nallet F, Douliez JP. 12-hydroxy 
stearic acid lipid tubes under various experimental conditions. Journal of Colloid and interface Science 2010 ; 341 : 38-47.

Fameau AL, Cousin F, Navailles L, Nallet F, Boué F, Doilez JP. Multiscale structural Characterizations of fatty acid multilayered tubes with a temperature-tunable diameter. The Journal of Physical Chemistry B 2011a ; 115 : 9033-9.

Fameau AL, Douliez JP, Boué F, Ott F, Cousin F. Adsorption of multilamellar tubes with a temperature tunable diameter at the air/water interface. Journal of Colloid and Interface Science 2011b ; 362 : 394-405.

Fameau AL, Houinsou Houssou B, Ventureira J, Navailles L, Nallet F, Novales B, Douliez JP. Self-assebly, foaming, and emulsifying properties of sodium alkyl carboxylate/guanidine hydrochloride aqueous mixtures. Langmuir 2011c ; 27 : 4505-13.

Fameau AL, Saint-Jalmes A, Cousin F, Houinsou-Houssou B, Novales B, Navailles
L, Nallet F, Emile J, Gaillard C, Boué F, Douliez JP. Smart Foams : Switching Reversibly between Ultrastable and Unstable Foams. Angewandte Chemie International Edition 2011d ; $50: 8264-8269$ and 11826.

Israelachvili J. Intermolecular and surface forces. Londres : Academic press Limited NW1 7DX, second edition, 1992.

Karleskind A. Manuel des corps gras. Paris : Lavoisier Edition, 1992.

Klein R, Touraud D, Kunz W. Choline carboxylate surfactants: biocompatible and highly soluble in water. Green chemistry 2008 ; 10 : 433-5.

Langevin D. Influence of interfacial rheology on foam and emulsion properties. Advances in Colloid and Interface Science $2000 ; 88$ : 209-22.

McBain JW, Salmon CS. Colloidal electrolytes, soap solutions and their constitution. Journal of the American Chemical Society $1920 ; 42$ : 426-60.
Novales B, Riaublanc A, Navailles L, Houinsou Houssou B, Gaillard C, Nallet F, Douliez JP. Self-assembly and foaming properties of fatty acid-lysine aqueous dispersions. Langmuir $2010 ; 26$ : 5329-34.

Saint-Jalmes A, Langevin D. Time evolution of aqueous foams: drainage and coarsening. Journal of Physics-condensed Matter 2002 ; 14 : 9397-412.

Saint-Jalmes A. Physical chemistry in foam drainage and coarsening. Soft Matter 2006 ; 2 : 836-49.

Vandeputte J. Les agro-tensioactifs. OCL $2012 ; 19: 133-7$.

Weaire D, Hutzler S. The Physics of Foams. Oxford : Clarenton Press, 1999.

Zana R. Partial phase behavior and micellar properties of tetrabutylammonium salts of fatty acids: Unusual solubility in water and formation of unexpectedly small micelles. Langmuir 2004 ; 20 : 5666-8. 